\title{
Application of intravenous lidocaine in patients undergoing operative hysteroscopy: a randomized, double-blind, controlled study
}

Huaxin Wang

Renmin Hospital of Wuhan University

\section{Xuan Peng}

Renmin Hospital of Wuhan University

Yeda Xiao

Renmin Hospital of Wuhan University

\section{Bo Zhao}

Renmin Hospital of Wuhan University

Liying Zhan ( 0 45123915@qq.com )

Renmin Hospital of Wuhan University https://orcid.org/0000-0002-7368-9932

Research article

Keywords: Lidocaine, hysteroscopic surgery, remifentanil, propofol

Posted Date: July 13th, 2019

DOI: https://doi.org/10.21203/rs.2.181/v2

License: (c) (1) This work is licensed under a Creative Commons Attribution 4.0 International License. Read Full License 


\section{Abstract}

Background The role of intraoperative intravenous lidocaine infusion has been previously evaluated for pain relief, inflammatory response, and post-operative recovery, including in endoscopic surgery. The present study is a randomized double-blinded trial in which we evaluated whether intravenous lidocaine infusion would reduce postoperative pain, propofol requirement and remifentanil consumption in patients undergoing hysteroscopy surgery. Methods Eighty-five patients scheduled to undergo elective operative hysteroscopy surgery under general anesthesia were randomized into two groups. Group $L$ included patients who received an intravenous lidocaine bolus $1.5 \mathrm{mg} / \mathrm{kg}$ over $3 \mathrm{~min}$ followed by a continuous infusion at the rate of $2 \mathrm{mg} / \mathrm{kg} / \mathrm{h}$ until surgery completed, and Group C received $0.9 \%$ normal saline solution at an equivalent rate. The depth of anesthesia was monitored using the Narcotrend, which was based on measurement of the patient's cerebral electrical activity. Primary outcome of the study was postoperative hypogastric pain evaluating by visual analogue scale (VAS). Secondary outcomes include propofol requirement and remifentanil requirement. Results VAS score of Group $L$ was significantly lower than Group $C$ at postoperative $0.5 \mathrm{~h}, 4 \mathrm{~h}$, respectively $(P<0.05)$, while no obvious difference was found at postoperative $24 \mathrm{~h}$. There was no difference between groups in propofol requirement, but Group $\mathrm{L}$ required less dosage of remifentanil than Group $C(P<0.05)$. Moreover, the incidence of throat pain was significantly lower in Group L. No adverse events associated with lidocaine was discovered. Conclusions Administration of intravenous lidocaine infusion as an adjuvant alleviated short-term postoperative hypogastric pain and throat pain, and reduced remifentanil requirement in patients undergoing operative hysteroscopy surgery.

\section{Introduction}

Operative hysteroscopy is routinely performed under general anesthesia (GA) in our hospital, which benefits both physicians and patients. First, GA can reduce potential difficulties of uterine access compared with local anesthesia[1]. Second, although a few studies reported that non-pharmacological approaches to pain control for hysteroscopy procedures (including distension method or physical stimuli) have contributed to improving patient satisfaction, hysteroscopy continues to be painful for a very high percentage of patients[2]. GA has been demonstrated to alleviate patients' experience of pain during the hysteroscopy. However, GA may be associated with postoperative pain, delayed recovery, and allergies.

Intraoperative lidocaine infusion has been widely accepted as an alternative to GA, as it can achieve opioid sparing, good pain relief, decreased post-operative nausea and vomiting, and excellent recovery[34]. Furthermore, recent studies showed that intravenous lidocaine infusion can reduce postoperative pain and opioid requirement among patients undergoing endoscopic submucosal dissection (ESD)[5]. Similar to $E S D$, operative hysteroscopy is an endoscopic procedure within natural lumens. However, to the best of our knowledge, the efficacy of intravenous lidocaine in operative hysteroscopy has not been fully known. Therefore, our study is to explore whether the administration of intravenous lidocaine can reduce postoperative pain, propofol requirement and remifentanil requirement in patients undergoing operative hysteroscopy. 


\section{Methods}

\section{Study population}

The study protocol was approved by the hospital ethics committee of Renmin Hospital of Wuhan University and was registered at http://www.chictr.org.cn (registration number: ChiCTR1800016857). Written informed consent was obtained from 85 patients with classification of American Society of Anesthesiologists (ASA) III undergoing operative hysteroscopy. Exclusion criteria include: less than 18 years old, hypersensitivity to lidocaine, only performing diagnostic hysteroscopy, chronic abuse of opioid or nonsteroidal anti-inflammatory drug, chronic pain, mis- or not understanding of oral information about the study, other severe systemic diseases, or serious surgical complications.

\section{Study group}

Patients were categorized into two study groups using a random number table method performed by an independent anesthetist (IA) not involved in the treatment or follow-up. The study drugs were prepared in syringes of identical appearance by the same IA as follows: $20-\mathrm{mL}$ syringe contained $1 \%$ lidocaine solution or $0.9 \%$ normal saline solution (for the bolus) and $50-\mathrm{mL}$ syringe contained $1 \%$ lidocaine solution or $0.9 \%$ normal saline solution (for the continuous infusion). The treatment and follow-up anesthetists were all blinded to patient assignment until analysis completion. The IA would cease the binding when the patient has abnormal conditions (such as serious complications) during the trial.

\section{Interventions}

Patients in the lidocaine group (Group L) received intravenous lidocaine with a bolus dose of $1.5 \mathrm{mg} / \mathrm{kg}$ over 3 minutes prior to anesthesia induction, then followed by continuous infusion at the rate of 2 $\mathrm{mg} / \mathrm{kg} / \mathrm{h}$ until the end of the surgery. Patients in the control group (Group C) received $0.9 \%$ normal saline solution at the equivalent rate. All patients were under the same anesthetic protocol without premedication. Standard monitoring included five-lead electrocardiography, oxygen saturation, noninvasive blood pressure and Narcotrend (NT) monitoring (MT Monitor Technik GmbH \& Co®KG, D24576 Bad Bramstedt, Germany). Anesthesia was induced with propofol $2.5 \mathrm{mg} / \mathrm{kg}$, then remifentanil 1.5 $\mu \mathrm{g} / \mathrm{kg}$ within one minute and followed by placing paraffin oil lubricated laryngeal mask (ALMA type, HangZhou FuShan Medical Appliances CO., LTD. China). Patients were given mechanical ventilation or converted to assisted ventilation when patients resumed spontaneous breathing. Anesthesia was maintained with continuous remifentanil and propofol infusion. Remifentanil was adjusted at a rate of 5$10 \mu \mathrm{g} / \mathrm{kg} / \mathrm{h}$, and the rate of propofol was adjusted according to the following Narcotrend Index (NTI) target values: during maintenance in a range of $37-64 ; 5$ min before the end of surgery in a range of 6579. In case of intraoperative patient movement, additional remifentanil $(1 \mu \mathrm{g} / \mathrm{kg})$ was injected immediately. 


\section{Outcomes and adverse events observation}

Mean blood pressure (MBP), heart rate (HR) and NTI were collected at 6 separate time points: before intravenous lidocaine (T0), before anesthesia induction (T1), 5 and 10 min after surgery (T2 and T3), the end of surgery (T4), the time to remove the laryngeal mask (T5). The total administered doses of remifentanil and propofol were recorded. Postoperative hypogastric pain at rest was evaluated at $0.5 \mathrm{~h}$ (T6), $4 \mathrm{~h}$ (T7), and $24 \mathrm{~h}$ (T8) after surgery by visual analogue scale (VAS, $0=$ no pain, $10=$ unbearable pain). In case of VAS $\geq 6,50 \mathrm{mg}$ flurbiprofen was given intravenously. The incidence of throat pain was assessed using VAS within 24 hours after surgery (any VAS $\geq 2$ considered to be sore throat). The incidence of postoperative nausea or vomiting was also recorded up to 24 hours after the procedure.

\section{Statistical analysis}

The primary outcome variable was postoperative hypogastric pain. A sample size was calculated based on the $0.5 \mathrm{~h}$ postoperative VAS score of 5 saline-administered patients (mean 2.3 and standard deviation 1.5) on the preliminary study. To demonstrate one mean difference of the VAS score at $0.5 \mathrm{~h}$ post operation between two groups with a two-tailed a of 0.05 and a power of $80 \%, 36$ candidates in each group were required. To compensate the dropout possibility for various reasons, we initially enrolled 85 patients in total for randomization. Statistical analysis was performed using GraphPad Prism version 6 (GraphPad Software Inc.). Quantitative variables were expressed as mean \pm standard deviation (SD); Categorical variables were presented as count (\%). The unpaired $t$ test and Fisher's exact test were used for statistical analysis. $P$ values less than 0.05 was considered to represent a statistically significant difference.

\section{Results}

The study flowchart was shown in Figure 1. Of 98 patients assessed for eligibility, 85 patients were enrolled and randomly assigned to two groups. Five patients ( 2 in Group L and 3 in Group C) were excluded from the analysis because only diagnostic hysteroscopy or uterine perforation was performed. Patient characteristics and surgical duration were similar between the groups (Table 1). For MAP, HR and $\mathrm{NTI}$ at the corresponding time points, no significant difference was found between the two groups (Figure 2).

Table 1 Baseline characteristics.

Table 2 Perioperative data.

The drug administration, VAS scores and postoperative events were demonstrated in Table 2. The total administered dose of propofol was lower in Group $L$, but not significantly different $(P=0.192)$. The total administered dose of remifentanil was $13 \%$ lower in Group L than in Group C (236.40 \pm 40.78 vs. 270.50 $\pm 47.38 \mu \mathrm{g}$, mean $\pm \mathrm{SD} ; P<0.001)$. No significant difference was identified in VAS at $24 \mathrm{~h}(T 8 ; P=0.111)$, 
but there was significant difference at postoperative $0.5 \mathrm{~h}$ and $4 \mathrm{~h}$ (T6 and T7) between the groups ( $P<$ $0.05)$. No patient was given flurbiprofen after surgery. Plasma levels of lidocaine were not analyzed, but the total dosage of lidocaine used in patients of Group L $(133.02 \pm 22.11 \mathrm{mg})$ was recorded. No lidocainerelated local anesthetic systemic toxicity was reported. Fewer patients in Group $L$ complained of throat pain $(22.5 \%$ vs. $47.5 \%, P=0.034)$. Both groups did not differ with respect to the incidence of nausea or vomiting $(P>0.05)$.

\section{Discussion}

Hysteroscopic procedures could be performed under mild sedation, local or general anesthesia. In this randomized, double-blind, placebo-controlled trial, we selected general anesthesia for the hysteroscopy, with intravenous lidocaine administered as a bolus of $1.5 \mathrm{mg} / \mathrm{kg}$ and then continuous infusion of 2 $\mathrm{mg} / \mathrm{kg} / \mathrm{h}$ during operative hysteroscopy. We found that this strategy could reduce the severity of earlier postoperative hypogastric pain and the incidence of throat pain. Moreover, lidocaine administration could also reduce the remifentanil requirement during operative hysteroscopy.

Intravenous infusion of lidocaine has shown effectiveness in controlling postoperative pain[6]. The analgesic mechanisms are multifactorial, including sodium channel blockade, reduction in spinal cord sensitivity and synergic effects with the general anesthetic agents[7]. The analgesic efficacy of intravenous lidocaine has been observed mainly in abdominal surgeries, including colectomy[8], gastrectomy[9], and cholecystectomy[10]. Moreover, Kim et al. confirmed the postoperative analgesic effects of intravenous lidocaine after ESD[5], an endoscopic surgical procedure of natural lumens similar to operative hysteroscopy. They also estimated that the analgesic effects of lidocaine were mainly exerted for visceral pain[5]. Therefore, we hypothesized that intravenous lidocaine would be beneficial for controlling visceral pain from operative hysteroscopy. In our study, administration of intravenous lidocaine resulted in reduction of earlier postoperative hypogastric pain intensity and less remifentanil consumption during operative hysteroscopy, which was consistent with a previous study reporting that intravenous lidocaine had a positive impact on pain scores in the early postoperative phase[11]. It was confirmed that the half-life of lidocaine was only 1.5-2 hours after bolus injection [12]. This may explain why analgesic effects were noted at earlier postoperative phase rather than at 24 hours in our study.

The NTI determined by the Narcotrend monitoring system is a dimensionless continuous variable scoring from 0 to 100 which reflects the depth of anaesthesia. Based on the NTI, the depth of anaesthesia is from stage $A$ (awake) to stage $F$ (very deep anaesthesia), with stage $D$ (37-64) indicating the routine depth of anaesthesia for surgery. Previous studies reported that intravenous lidocaine had a propofol-sparing effect during GA [13-14]. In this study, we ensured that each patient was at the constant anesthetic depth during surgery by Narcotrend monitor. Our results showed that intravenous use of lidocaine could reduce the amount of propofol dosage, but there was no significant difference. This is probably due to the short hysteroscopic duration and hence insufficient observation time. 
Remifentanil is a preferred drug for endoscopy because of its rapid onset and offset of action, and minimal adverse effects on cardiovascular and respiratory parameters[15]. To observe postoperative earlier analgesic effect of intravenous lidocaine, another advantage of remifentanil is its ability to avoid interference of using other opioid drugs. Administration of intravenous lidocaine was shown to have an opioid sparing effect during GA, including fentanyl[16], sufentanil[17] and morphine[18]. In a recent study, the remifentanil sparing effect of intravenous lidocaine in the intraoperative period was found [19], which was consistent with our findings. However, another study reported that perioperative intravenous lidocaine infusion had no significant effect on remifentanil requirement during hypotensive anesthesia for elective transsphenoidal endoscopic hypophyseal adenoma excision procedure [20]. This difference was likely due to the distinct types of surgery performed and different regimes of anesthetic management.

We used the laryngeal mask for mechanical ventilation or assisted ventilation to assure the respiratory safety in this study. In addition, the placement of the laryngeal mask could also provide us another way to observe the analgesic effect of lidocaine. Administration of intravenous lidocaine reduced the incidence of throat pain caused by placing the laryngeal mask from $47.5 \%$ to $22.5 \%$ in this study. Our observation was in keeping with several studies showing that administration of IV lidocaine was effective in reducing throat pain after endoscopic submucosal dissection[5] and postoperative sore throat caused by tracheal intubation[21]. Last, we did not see any significant differences in the incidence of postoperative nausea or vomiting, which is consistent with previous reports [22-23].

There are some limitations in our study. We did not measure the plasma levels of lidocaine in our patients as it is not available in our institution. However, the protocol of administering a loading dose followed by continuous IV infusion of lidocaine during GA has been used in several previous centers and was reported to be well below the toxic level[24-25]. Another limitation is the small number of patients. A larger-scale trial will provide further details to validate our findings.

In conclusion, Intravenous lidocaine infusion for operative hysteroscopy can alleviate earlier postoperative hypogastric pain and throat pain, and decreased remifentanil consumption.

\section{Abbreviations}

ASA = American Society of Anesthesiologists, $E S D=$ endoscopic submucosal dissection, $G A=$ general anesthesia, $\mathrm{HR}$ = heart rate, $\mathrm{IA}=$ independent anesthetist, $\mathrm{MBP}=$ mean blood pressure, $\mathrm{NT}=\mathrm{Narcotrend}$, $\mathrm{NTI}=$ Narcotrend Index, $\mathrm{SD}=$ standard deviation

\section{Declarations}

\section{Ethics approval and consent to participate}

The study protocol was approved by the hospital ethics committee of Renmin Hospital of Wuhan University. Written informed consent was obtained from all patients. 


\section{Consent for publication}

Not Applicable.

\section{Availability of data and material}

All data and material are kept by the corresponding author and are available in paper for any further question.

\section{Competing interests}

The authors have no conflicts of interest to disclose.

\section{Funding}

This study was supported by the China Scholarship Council (No. 201706275133) and the Basic Scientific Research Foundation of Central Universities (No. 2042017kf0144).

\section{Author contributions}

Huaxin Wang and Liying Zhan designed this study together. Huaxin Wang, Xuan Peng and Liying Zhan performed the statistical analysis and drafted the protocol. Huaxin Wang and Liying Zhan was responsible for the writing revision.

Data curation: Huaxin Wang, Liying Zhan.

Formal analysis: Huaxin Wang, Xuan Peng, Liying Zhan.

Investigation: Huaxin Wang, Xuan Peng, Yeda Xiao, Bo Zhao.

Methodology: Xuan Peng, Bo Zhao.

Project administration: Yeda Xiao, Bo Zhao, Liying Zhan.

Writing - original draft: Huaxin Wang, Xuan Peng.

Funding acquisition: Huaxin Wang.

Writing - review and editing: Huaxin Wang, Xuan Peng, Liying Zhan.

\section{Acknowledgements}


The authors thank all the patients and staff of this study for their important contributions.

\section{References}

1. Munro M. G., Brooks P. G. Use of local anesthesia for office diagnostic and operative hysteroscopy. J Minim Invasive Gynecol. 2010; 17:709-718.

2. Amer-Cuenca J. J., Marin-Buck A., Vitale S. G., La Rosa V. L., Caruso S., Cianci A., Lison J. F. Nonpharmacological pain control in outpatient hysteroscopies. Minim Invasive Ther Allied Technol. 2019; 22: 1-10.

3. Lauwick S., Kim D. J., Michelagnoli G., Mistraletti G., Feldman L., Fried G., Carli F. Intraoperative infusion of lidocaine reduces postoperative fentanyl requirements in patients undergoing laparoscopic cholecystectomy. Can J Anaesth. 2008; 55:754-760.

4. Kranke P., Jokinen J., Pace N. L., Schnabel A., Hollmann M. W., Hahnenkamp K., Eberhart L. H., Poepping D. M., Weibel S. Continuous intravenous perioperative lidocaine infusion for postoperative pain and recovery. Cochrane Database Syst Rev. 2015; 16: CD009642.

5. Kim J. E., Choi J. B., Koo B. N., Jeong H. W., Lee B. H., Kim S. Y. Efficacy of Intravenous Lidocaine During Endoscopic Submucosal Dissection for Gastric Neoplasm: A Randomized, Double-Blind, Controlled Study. Medicine (Baltimore). 2016; 95: e3593.

6. Estebe J. P. Intravenous lidocaine. Best Pract Res Clin Anaesthesiol. 2017; 31: 513-521.

7. Lauretti G. R. Mechanisms of analgesia of intravenous lidocaine. Rev Bras Anestesiol. 2008; 58: 280286.

8. Lee M. W., Or D. Y., Tsang A. C., Ng D. C., Chen P. P., Cheung M. H., Li R. S., Leong H. T. Intravenous lignocaine infusion facilitates acute rehabilitation after laparoscopic colectomy in the Chinese patients. Hong Kong Med J. 2017; 23: 441-445.

9. Yon J. H., Choi G. J., Kang H., Park J. M., Yang H. S. Intraoperative systemic lidocaine for pre-emptive analgesics in subtotal gastrectomy: a prospective, randomized, double-blind, placebo-controlled study. Can J Surg. 2014; 57: 175-182.

10. Zhao J. B., Li Y. L., Wang Y. M., Teng J. L., Xia D. Y., Zhao J. S., Li F. L. Intravenous lidocaine infusion for pain control after laparoscopic cholecystectomy: A meta-analysis of randomized controlled trials. Medicine (Baltimore). 2018;97: e9771.

11. Kranke P., Jokinen J., Pace N. L., Schnabel A., Hollmann M. W., Hahnenkamp K., Eberhart L. H., Poepping D. M., Weibel S. Continuous intravenous perioperative lidocaine infusion for postoperative pain and recovery. Cochrane Database Syst Rev. 2015;16:CD009642. 
12. de Oliveira C. M., Issy A. M., Sakata R. K. Intraoperative intravenous lidocaine. Rev Bras Anestesiol. 2010; 60: 325-333.

13. Xu S. Q., Li Y. H., Wang S. B., Hu S. H., Ju X., Xiao J. B. Effects of intravenous lidocaine, dexmedetomidine and their combination on postoperative pain and bowel function recovery after abdominal hysterectomy. Minerva Anestesiol. 2017; 83: 685-694.

14. Cui W., Li Y., Li S., Wang R., Li J. Systemic administration of lidocaine reduces morphine requirements and postoperative pain of patients undergoing thoracic surgery after propofol-remifentanil-based anaesthesia. Eur J Anaesthesiol. 2010; 27:41-46.

15. Stroumpos C., Manolaraki M., Paspatis G. A. Remifentanil, a different opioid: potential clinical applications and safety aspects. Expert Opin Drug Saf. 2010; 9: 355-364.

16. Ho MLJ, Kerr S. J., Stevens J. Intravenous lidocaine infusions for 48 hours in open colorectal surgery: a prospective, randomized, double-blinded, placebo-controlled trial. Korean J Anesthesiol. 2018; 71: 57-65.

17. Sloan T. B., Mongan P., Lyda C., Koht A. Lidocaine infusion adjunct to total intravenous anesthesia reduces the total dose of propofol during intraoperative neurophysiological monitoring. J Clin Monit Comput. 2014; 28: 139-147.

18. Ibrahim A., Aly M., Farrag W. Effect of intravenous lidocaine infusion on long-term postoperative pain after spinal fusion surgery. Medicine (Baltimore). 2018; 97: e229.

19. Nakhli M. S., Kahloul M., Guizani T., Zedini C., Chaouch A., Naija W. Intravenous lidocaine as adjuvant to general anesthesia in renal surgery. Libyan J Med. 2018; 13: 1433418.

20. Uzun S., Yuce Y., Erden A., Aypar U. Impact of perioperative lidocaine infusion and bis monitorization on remifentanil dosage in hypotensive anesthesia. Eur Rev Med Pharmacol Sci. 2014; 18: 559-565.

21. Tanaka Y., Nakayama T., Nishimori M., Tsujimura Y., Kawaguchi M., Sato Y. Lidocaine for preventing postoperative sore throat. Cochrane Database Syst Rev. 2015; 14: CD004081.

22. Gholipour Baradari A., Firouzian A., Hasanzadeh Kiabi F., Emami Zeydi A., Khademloo M., Nazari Z., Sanagou M., Ghobadi M., Fooladi E. Bolus administration of intravenous lidocaine reduces pain after an elective caesarean section: Findings from a randomised, double-blind, placebo-controlled trial. J Obstet Gynaecol. 2017;37: 566-570.

23. Farag E., Ghobrial M., Sessler D. I., Dalton J. E., Liu J., Lee J. H., Zaky S., Benzel E., Bingaman W., Kurz A. Effect of perioperative intravenous lidocaine administration on pain, opioid consumption, and quality of life after complex spine surgery. Anesthesiology. 2013;119: 932-940.

24. Kaba A., Laurent S. R., Detroz B. J., Sessler D. I., Durieux M. E., Lamy M. L., Joris J. L. Intravenous lidocaine infusion facilitates acute rehabilitation after laparoscopic colectomy. Anesthesiology. 2007; 
106: 11-18, 5-6.

25. Bazin P., Padley J., Ho M., Stevens J., Ben-Menachem E. The effect of intravenous lidocaine infusion on bispectral index during major abdominal surgery. J Clin Monit Comput. 2018;32:533-539.

\section{Tables}

Table 1 Baseline characteristics.

\begin{tabular}{|c|c|c|c|c|}
\hline & $\begin{array}{l}\text { Group L } \\
(\mathrm{n}=40)\end{array}$ & $\begin{array}{l}\text { Group C } \\
(n=40)\end{array}$ & $\begin{array}{l}\text { Statistics (unpaired } t \text { test or Fisher's } \\
\text { exact) }\end{array}$ & $\begin{array}{l}p- \\
\text { Value }\end{array}$ \\
\hline Age (years) & $\begin{array}{l}31.38 \pm \\
7.37\end{array}$ & $32.85 \pm 7.73$ & $t=0.837$ & 0.385 \\
\hline Weight (kg) & $53.20 \pm 8.02$ & $\begin{array}{l}55.10 \pm \\
10.61\end{array}$ & $t=0.904$ & 0.369 \\
\hline Height (cm) & $\begin{array}{l}167.00 \pm \\
7.90\end{array}$ & $\begin{array}{l}168.10 \pm \\
8.50\end{array}$ & $t=0.814$ & 0.418 \\
\hline $\begin{array}{l}\text { ASA physical status, } \\
n(\%)\end{array}$ & & & & 0.642 \\
\hline ૫ & $24(60 \%)$ & 27 (67.5\%) & & \\
\hline$\square$ & $16(40 \%)$ & 13 (32.5\%) & & \\
\hline $\begin{array}{l}\text { Duration of surgery } \\
\text { (min) }\end{array}$ & $\begin{array}{l}29.83 \pm \\
9.53\end{array}$ & $27.45 \pm 7.46$ & $t=1.241$ & 0.218 \\
\hline
\end{tabular}

Values are shown as mean \pm SD or number of patients (proportion). ASA = American Society of Anesthesiologists.

Table 2 Perioperative data. 


\begin{tabular}{lllll} 
& $\begin{array}{l}\text { Group } \mathrm{L} \\
(\mathrm{n}=40)\end{array}$ & $\begin{array}{l}\text { Group } \mathrm{C} \\
(\mathrm{n}=40)\end{array}$ & $\begin{array}{l}\text { Statistics (unpaired } t \text { test or Fisher's } \\
\text { exact) }\end{array}$ & $\begin{array}{l}p \text { - } \\
\text { Value }\end{array}$ \\
\hline Total propofol $(\mathrm{mg})$ & $\begin{array}{l}237.20 \pm \\
49.30\end{array}$ & $\begin{array}{l}251.90 \pm \\
50.45\end{array}$ & $\mathrm{t}=1.316$ & 0.192 \\
\hline $\begin{array}{l}\text { Total remifentanil } \\
(\mu \mathrm{g})\end{array}$ & $\begin{array}{l}236.40 \pm \\
40.78\end{array}$ & $\begin{array}{l}270.50 \pm \\
47.38\end{array}$ & $\mathrm{t}=3.452$ & 0.001 \\
\hline VAS & & & & \\
\hline T6 & $1.83 \pm 1.24$ & $2.43 \pm 1.10$ & $\mathrm{t}=2.285$ & 0.025 \\
\hline T7 & $1.98 \pm 0.95$ & $2.63 \pm 1.23$ & $\mathrm{t}=2.643$ & 0.010 \\
\hline T8 & $0.90 \pm 0.81$ & $1.20 \pm 0.85$ & $\mathrm{t}=1.612$ & 0.111 \\
\hline Throat pain, $\mathrm{n}(\%)$ & $9(22.5 \%)$ & $19(47.5 \%)$ & & 0.034 \\
\hline $\begin{array}{l}\text { Nausea or vomiting, } \\
\mathrm{n}(\%)\end{array}$ & $4(10 \%)$ & $9(22.5 \%)$ & & 0.225 \\
\hline
\end{tabular}

Values are presented as mean $\pm S D$ or number of patients (proportion). VAS $=$ visual analogue scale $(0=$ no pain, $10=$ unbearable pain). $\mathrm{T} 6=0.5 \mathrm{~h}$ after surgery, $\mathrm{T} 7=4 \mathrm{~h}$ after surgery, $\mathrm{T} 8=24 \mathrm{~h}$ after surgery.

\section{Figures}




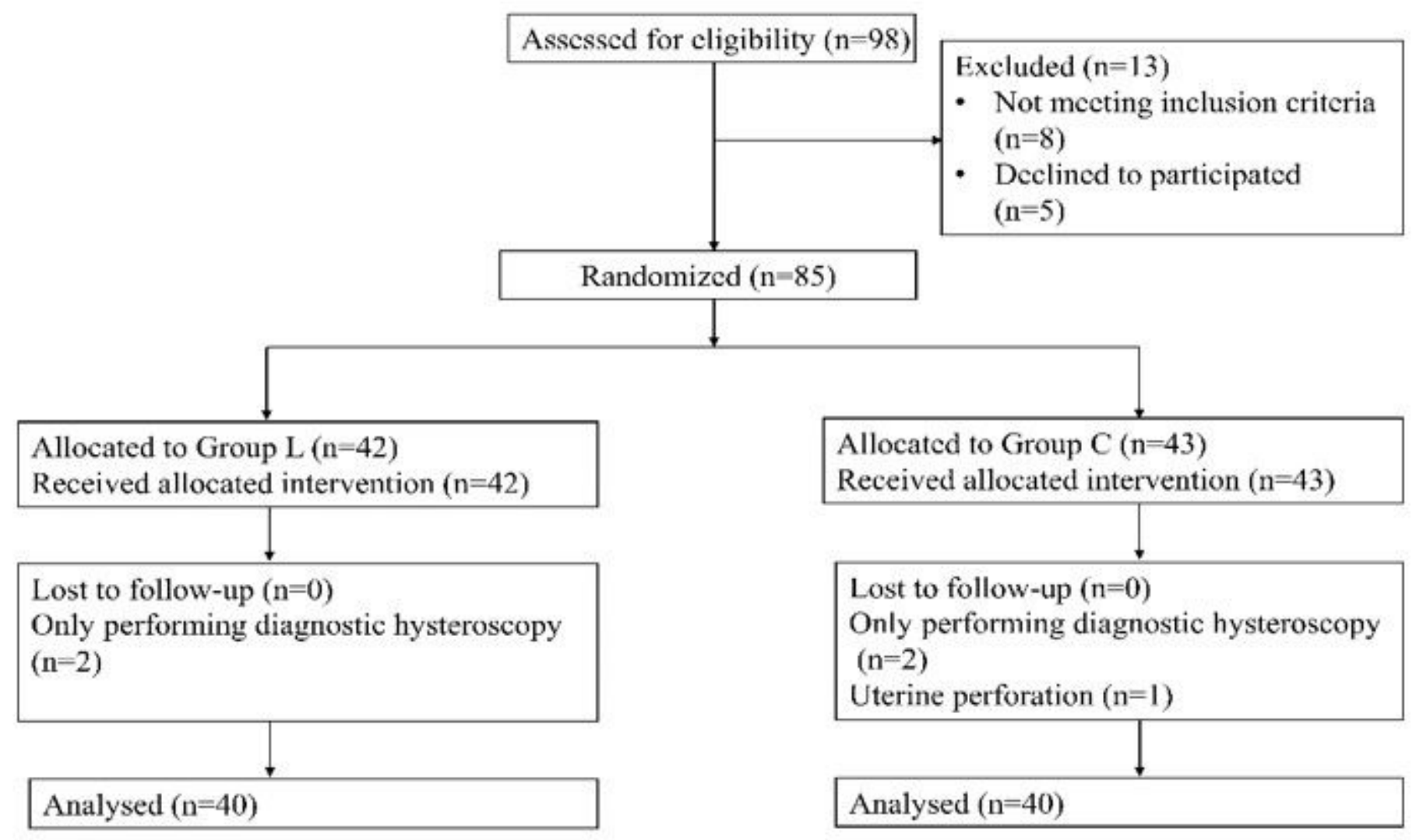

Figure 1

Flow diagram of patient recruitment.

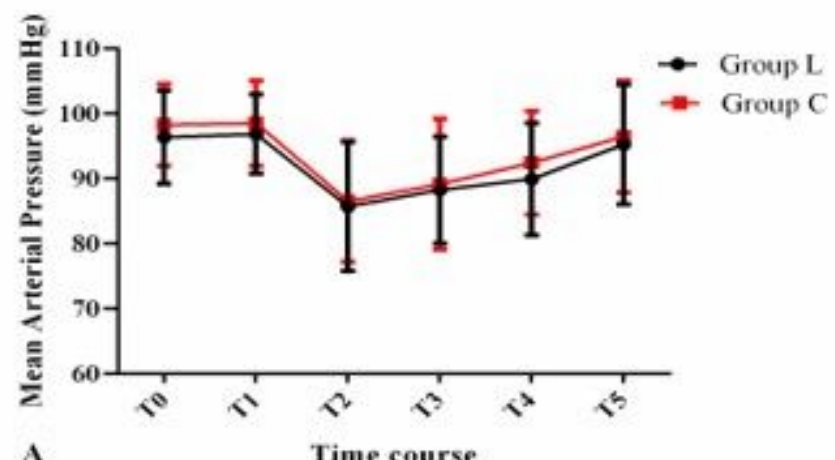

A

Time course
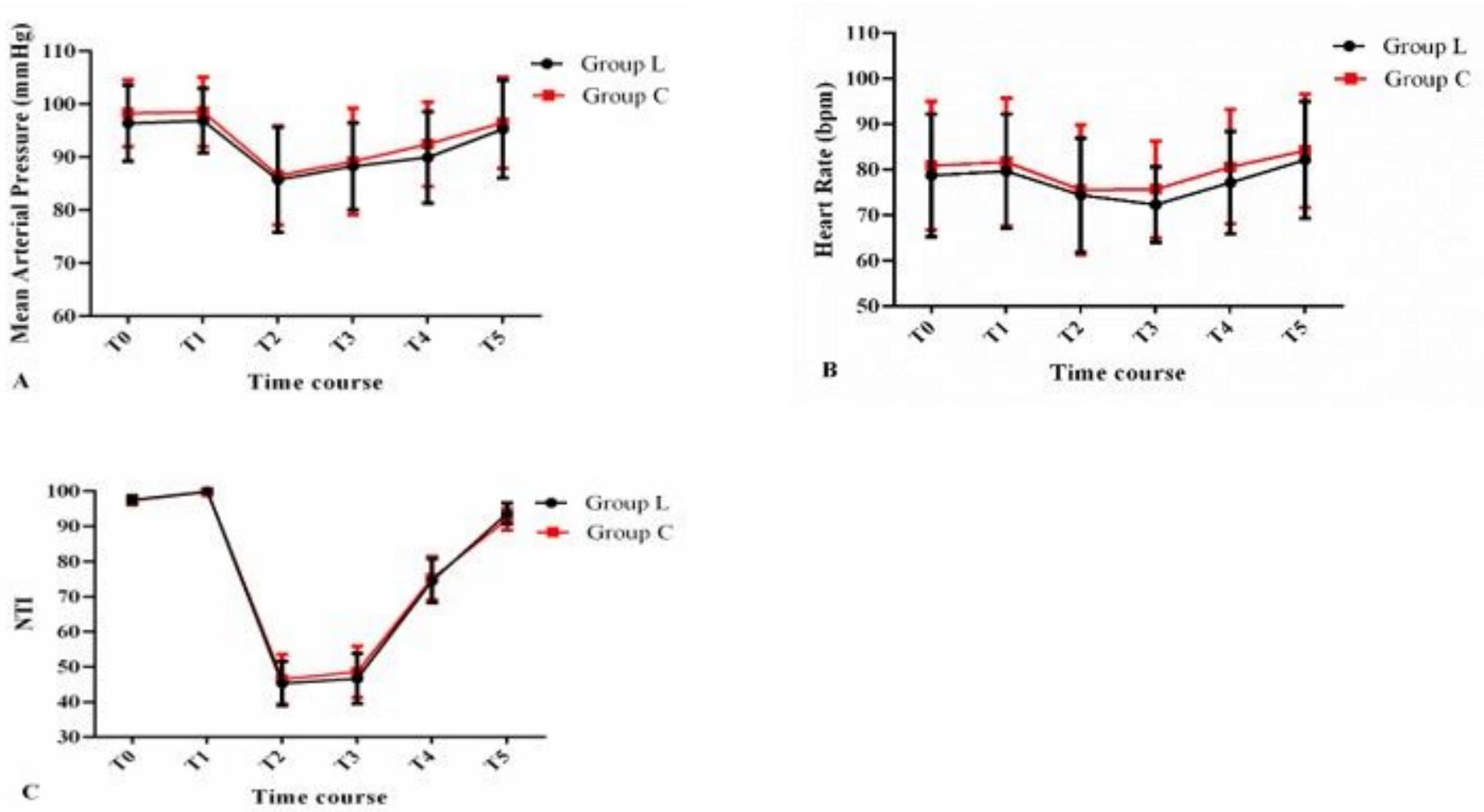

Figure 2 
Changes in (A) mean blood pressure, $(\mathrm{B})$ heart rate and (C) NTI. T0 $=$ before intravenous lidocaine, $\mathrm{T} 1=$ before anesthesia induction, $\mathrm{T} 2=5 \mathrm{~min}$ after surgery, $\mathrm{T} 3=10$ minutes after surgery, $\mathrm{T} 4=$ the end of the surgery, $\mathrm{T} 5=$ the time to remove the laryngeal mask. NTI = Narcotrend Index. Data are expressed as mean \pm SD.

\section{Supplementary Files}

This is a list of supplementary files associated with this preprint. Click to download.

- Throatpain.pzfx

- consort2010checklist.pdf

- HRT5.pzfx

- MAPdifferenttime.pzfx

- MAPT0.pzfx

- Height.pzfx

- HRdifferenttime.pzfx

- HRT0.pzfx

- HRT2.pzfx

- HRT3.pzfx

- Durationofsurgery.pzfx

- ASA.pzfx

- Age.pzfx

- NTIT5.pzfx

- NTIT1.pzfx

- VAST8.pzfx

- Data.rar

- HRT4.pzfx

- Totaldosageoflidocaine.pzfx

- NTIT2.pzfx

- Totalpropofol.pzfx

- Totalremifentanil.pzfx

- VAST6.pzfx

- VAST7.pzfx

- NTIdifferenttime.pzfx

- NTIT0.pzfx

- NTIT3.pzfx

- HRT1.pzfx 
- NTIT4.pzfx

- MAPT1.pzfx

- MAPT2.pzfx

- MAPT3.pzfx

- MAPT4.pzfx

- Weight.pzfx

- Nauseaorvomiting.pzfx

- MAPT5.pzfx 